\title{
On the dynamics of cutting arc plasmas: the role of the power supply ripple
}

\author{
L. Prevosto ${ }^{1, *}$, B. Mancinelli ${ }^{1}$, and H. Kelly ${ }^{1,2}$ \\ ${ }^{1}$ Grupo de Descargas Eléctricas, Departamento Ingeniería Electromecánica \\ Facultad Regional Venado Tuerto (UTN) Laprida 651 (2600), Venado Tuerto (Santa Fe), Argentina \\ ${ }^{2}$ Instituto de Física del Plasma (CONICET), Departamento de Física \\ Facultad de Ciencias Exactas y Naturales (UBA) \\ Ciudad Universitaria Pab. I, (1428) Buenos Aires, Argentina \\ *corresponding author, E-mail: prevosto@waycom. com.ar
}

\begin{abstract}
The power sources used in cutting arc torches are usually poorly stabilized and have a large ripple factor. The strong oscillatory components in the voltage and arc current produce in turn, large fluctuations in the plasma quantities. Experimental observations on the dynamics of the nonequilibrium plasma inside the nozzle of a 30 A oxygen cutting torch with a $7 \%$ ripple level of its power source are reported in this work. The observed electron temperature (mean value $\approx 5400 \mathrm{~K})$ shows a rms deviation $\approx 5 \%( \pm$ $300 \mathrm{~K})$, which is of the order of the arc voltage ripple level. A considerable different situation occurs with the plasma density (mean value $\approx 3 \times 10^{19} \mathrm{~m}^{-3}$ ). In this case the rms deviation was $\approx 75 \%$, much greater than that of the electron temperature.
\end{abstract}

\section{Introduction}

The plasma cutting process is characterized by a transferred electric arc that is established between a cathode, which is a part of the cutting torch, and a work-piece (the metal to be cut) acting as the anode [1]. In order to obtain a highquality cut, the plasma jet must be as collimated as possible (i.e., it must have high power density). To this end, the transferred arc is constricted by a metallic tube (a nozzle) with a small inner diameter (of the order of one millimeter). A vortex-type high-pressure flow is forced through the nozzle to provide arc stability and to protect its inner wall.

Time fluctuations in thermal plasma direct-current (dc) devices are important for any technological application. A special effort has been made by several researchers (see for instance Ref. [2] to [4]) both from an experimental or a numerical points of view, to understand the dynamics of the plasma flow inside non-transferred arc torches (like spraying torches). In such torches, large plasma jet fluctuations arise either from ripple in the direct-current power supply, or random arc root movement at the anode, or combined effects of gas dynamic and electromagnetic instabilities causing cold gas entrainment. On the other hand, it is implicitly assumed in most of the experimental or numerical works in transferred arc torches (like cutting torches), that the plasma is steady [1]. However, the power sources used in such plasma torches are usually poorly stabilized and have a large ripple factor (with root-meansquare - rms- deviations that vary between $\approx 5$ to $10 \%$ of the mean voltage). This is due to the fact that the torch currents are of the order of $100 \mathrm{~A}$, which difficult an effective filtering of the ripple. If a 3-phase transductor type of power supply is used, then the fundamental ripple frequency is $150 \mathrm{~Hz}$ and if 3-phase silicon controlled rectifier based power supply is used, then the ripple frequency is $300 \mathrm{~Hz}$. The strong oscillatory components in the voltage and arc current should produce in turn, large fluctuations in the plasma quantities that vary at the ripple frequency.

In this work, experimental observations on the plasma dynamics inside the nozzle of a cutting torch are reported. It is employed a technique previously developed in our laboratory consisting in using the nozzle as a large-sized Langmuir probe [5].

\section{Experimental set-up}

The experiment was carried out using an oxygen highenergy density cutting torch. It consisted of a cathode centered above an orifice in a converging-straight copper nozzle without liquid cooling. The cathode was made of copper ( $7 \mathrm{~mm}$ in diameter) with a hafnium tip $(1.5 \mathrm{~mm}$ in diameter) inserted at the cathode center. A flow of oxygen gas cooled the cathode and the nozzle and was also employed as the plasma gas. The gas passed through a swirl ring to provide arc stability. The nozzle consisted in a converging-straight bore (with a bore radius $R_{N}=0.5 \mathrm{~mm}$ and a length $L_{N}=4.5 \mathrm{~mm}$ ) in a copper holder surrounding the cathode (with a separation of $0.5 \mathrm{~mm}$ between the holder and the cathode surface). To avoid plasma contamination by metal vapors from the anode, a rotating steel disk was used as the anode, with its upper surface located at $6 \mathrm{~mm}$ from the nozzle exit. A well-stabilized arc column was obtained, with the arc root sliding on the disk lateral surface. It was found that this surface resulted completely not melted (thus, practically no metal vapors from the anode were present in the arc). More details on the 
experimental configuration can be found elsewhere [6]. A 3-phase transductor power supply with a voltage ripple level of $\approx 7 \%$ and a fundamental frequency of $150 \mathrm{~Hz}$ was used to run the torch. The measured torch operating conditions were: arc current $30 \mathrm{~A}$ (rms), gas mass flow rate $0.39 \mathrm{~g} \mathrm{~s}^{-1}$ and torch chamber pressure $p_{c h}=0.6 \mathrm{MPa}$. For the voltage measurements, the reference electrode was the anode (grounded) and the cathode voltage resulted in -145 $\mathrm{V}$ (rms).

As previously quoted, the nozzle was used as a large sized Langmuir probe. Note that the necessary condition for a comprehensive use of a Langmuir probe (that is: the plasma should not be perturbed sufficiently far away from the probe surface) is accomplished in this case, since the nozzle-probe behaves as a natural boundary to the arc. The nozzle biasing circuit is shown in Fig. 1. Different nozzleanode voltage values $\left(V_{N}\right)$ were obtained using a highimpedance rheostat ( $2 \mathrm{k} \Omega$ of total resistance), connected between the cathode and the anode of the arc, while the nozzle current $\left(i_{N}\right)$ was calculated from the voltage drop through a small resistance $R_{0}$. In practice, the nozzle was always biased close to the cathode voltage. Both the $V_{N}$ and $i_{N}$ waveforms were registered by using a two-channel digital oscilloscope (Tektronix TDS 1002 B) with a sampling rate of $500 \mathrm{MS} / \mathrm{s}$ and an analogical bandwidth of $60 \mathrm{MHz}$.

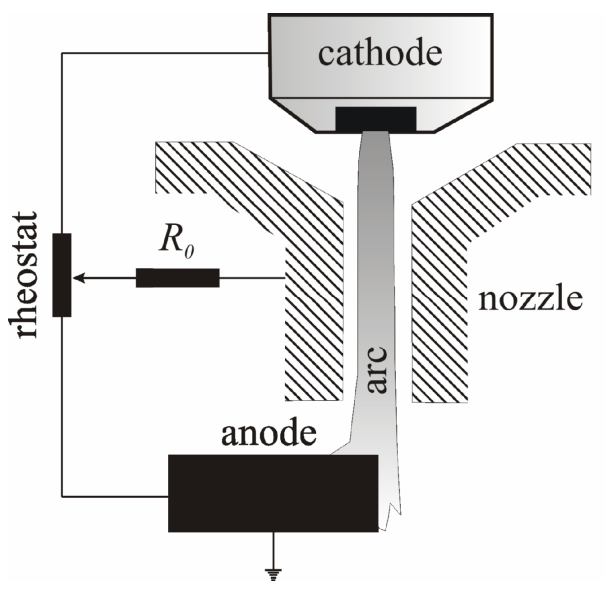

Figure 1: Schematic of the nozzle biasing circuit.

Finally, a set of sweeping electrostatic probes [6] was used to determine the floating potential close to the nozzle exit (at $0.5 \mathrm{~mm}$ ). The plasma potential value $\left(V_{p}\right)$ at the nozzle exit was then obtained from the floating experimental value taking into account an electric field strength of $\approx 7 \mathrm{~V} / \mathrm{mm}$ in the external part of the arc [7] and the voltage drop between the plasma and the floating potentials (which is proportional to the plasma electron temperature). In the case we are considering, we will show later that the electron temperature is relatively low (about $5000-6000 \mathrm{~K}$ at the arc border) and hence such difference results of only about $5 \mathrm{~V}$ [8].

\section{Results and discussion}

A typical ion current $\left(i_{N}\right)$ waveform for a rms nozzle voltage value close to the cathode one is shown in Fig. 2. The result of performing Fourier analysis on the ion current signal is also shown. The rms value of the oscillatory component reaches $\approx 52 \%$ of the mean value with a fundamental frequency $(f)$ of $150 \mathrm{~Hz}$ (i.e., the ripple frequency). A marked spike of short duration (of the order of $100 \mu \mathrm{s}$ ) which actually presents a complex fine structure associated with the non-destructive double-arcing phenomenon is clearly recognized in this figure [9].

From the floating potential measurement close to the nozzle exit, a plasma potential mean value of $\approx-20 \mathrm{~V}$ (with a rms deviation of $7 \%$ ) was obtained for the given arc operating conditions.

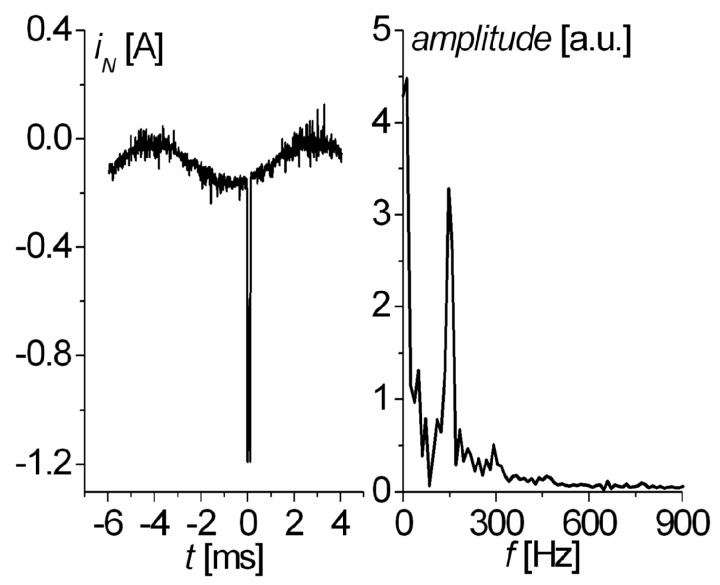

Figure 2: Typical ion current waveform. The FFT analysis is also shown.

In what follows, it will be presented a physical interpretation of the ion current signal taking into account the ripple component. This interpretation is based on the use of a physical model for the space-charge layer between the arc plasma and the nozzle wall. Since the temporal variations introduced by the ripple are very slow as compared with the characteristic times in the layer (electron plasma frequency, transit time of sound perturbations along the layer, etc.), a previously developed steady model $[5,10]$ will be adopted, with the additional assumption that the plasma quantities follows instantaneously the arc current and arc voltage variations.

At the vicinities of the nozzle wall the electron temperature $\left(T_{e}\right)$ is well decoupled from the gas temperature (close to the temperature of the nozzle wall $T_{h} \approx 1000 \mathrm{~K}$ ). The composition of this non-equilibrium plasma was calculated by using the generalized Saha-equation [11]

$$
\frac{n^{2}}{n_{n}}=2 \frac{Q_{i}}{Q_{0}}\left(\frac{2 \pi m k T_{e}}{h^{2}}\right)^{3 / 2} \exp \left(-\frac{E_{I}}{k T_{e}}\right)
$$

plus the equation of state

$$
\frac{p}{k}=T_{e} n+T_{h}\left(n+n_{n}\right)
$$


(where $n$ is the plasma density, $n_{n}$ is the neutral particle density, $Q_{i}$ and $Q_{o}$ are the statistical weights of oxygen ions and atoms, $m$ is the electron mass, $k$ is the Boltzmann's constant, $h$ is the Planck's constant, $E_{I}$ is the ionization energy of the oxygen atoms, and $p$ is the pressure).

The ion current collected by the nozzle was calculated by integrating the ion flux evaluated at the edge of the plasma-layer boundary

$$
\left.i_{N}=2 \pi R_{N} e \int_{z=0}^{z=L_{N}} \Gamma_{+}\right)_{s} d z
$$

where $\left.\Gamma_{+}\right)_{s}=n_{s} v_{+s}$ ( $e$ is the electron charge and $z$ is the coordinate directed along the nozzle wall). Due to the colisional regime of the layer $[5,10]$ the ion entrance velocity $v_{+\mathrm{s}}$ is lower than the Bohm velocity in a factor of $\left(\lambda_{+} / \lambda_{D s}\right)^{1 / 2}$ [12]. (Here $\lambda_{+}$is the colisional ion mean free path and $\lambda_{D s}$ is the electron Debye length at the layer entrance)

The thickness of such a space-charge layer was approximated as [13]

$$
D \approx 2.2 \times 10^{4} \Delta V^{3 / 5} n^{-1 / 2} T_{e}^{-1 / 10}
$$

where all the physical variables are given in MKS units ( $\Delta V \equiv V_{N}-V_{p}$ ). Eq. (4) allows to determine $D$ at the nozzle exit, since at this position $V_{p}$ is known from the floating potential measurements.

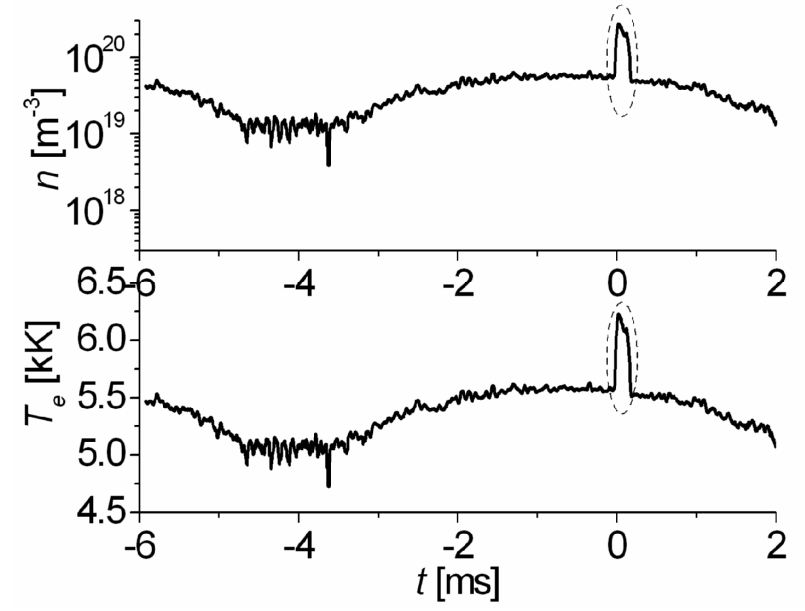

Figure 3: The electron temperature and the plasma density calculated from the experimental results showed in Fig. 2.

The formulation also includes the following assumptions: (i) a linear variation of the pressure $(p)$ along the nozzle (where $p(0)=p_{c h}$ and $p\left(L_{N}\right)=0.1 \mathrm{MPa}$ ); (ii) at the adjacency of the nozzle wall $T_{e}$ only depends on the radial coordinate; and (iii) a constant value of the layer thickness along the nozzle. Assumptions (i) and (ii) have been shown to be valid in cutting torches (e.g., [14]). Concerning the assumption (iii), it has been shown to be valid in cutting torches operated at low gas flow rates [5]. With all these considerations, Eqs. (1), (2), and (3) constituted a closed system that allowed to obtain $n_{n}, n$, and $T_{e}$ at the plasma- layer boundary for given values of $p(z)$ and $i_{N}$. In particular, the integral of Eq. (3) was approximated by a nine-term polynomial using the well-known Chebyshev formula. Finally, the $D$ value was obtained from the decoupled Eq. (4) in terms of the previously obtained plasma quantities.

Fig. 3 shows the oscillations of $T_{e}$ and the plasma density $n$ corresponding to the experimental results showed in Fig. 2. Note that the $T_{e}$ and $n$ values during the spike development cannot be taken with confidence since the assumptions employed to perform the inversion could not be valid (especially Eq. (4), which assumes that the particle creation or destruction processes can be ignored) at these times. The observed electron temperature (mean value $\approx$ $5400 \mathrm{~K})$ shows a rms deviation $\approx 5 \%( \pm 300 \mathrm{~K})$, which is of the order of the arc voltage ripple level. A considerable different situation occurs with $n$. In this case the rms deviation was $\approx 75 \%$, much greater than that of $T_{e}$. This is expected because in this scenario Eq. (1) approximately gives $n \propto T_{e}^{\alpha}$ (with $\alpha \approx 15$ ).

\section{Conclusions}

The non-equilibrium plasma inside the nozzle is far from the steady state in time, in contrast to what is frequently assumed. The ripple of the power source was identified as the main oscillation source. Arc electric oscillations due to the power source ripple exert strong influence on the characteristics of the plasma inside the nozzle of a cutting torch. In particular, the most sensitive quantity is the plasma density which shows a rms deviation $\approx 75 \%$, much greater than that of the electron temperature of the order of the arc voltage ripple level $\approx 7 \%$. This is expected because the plasma density grows strongly with the electron temperature for relatively low values $\approx 5000-6000 \mathrm{~K}$ of this last quantity.

\section{Acknowledgements}

This work was supported by grants from CONICET (PIP 5378) and Universidad Tecnológica Nacional (PID 1389). L $\mathrm{P}$ and $\mathrm{H} \mathrm{K}$ are members of the CONICET.

\section{References}

[1] V. A. Nemchinsky and W. S. Severance, What we know and what we do not know about plasma arc cutting, $J$. Phys. D: Appl. Phys. 39: R423-438, 2006.

[2] V. Rat and J. F. Coudert, Pressure and arc voltage coupling in dc plasma torches: Identification and extraction of oscillation modes, J. Appl. Phys. 108: 043304, 2010.

[3] J. F. Coudert and V. Rat, Influence of configuration and operating conditions on the electric arc instabilities of a plasma spray torch: role of acoustic resonance, J. Phys. D: Appl. Phys. 41: 205208, 2008.

[4] S. Ghorui and A. K. Das, Origin of fluctuations in atmospheric pressure arc plasma devices, Phys. Rev. E 69: 026408, 2004.

[5] L. Prevosto, H. Kelly and B. Mancinelli, On the physical origin of the nozzle characteristic and its connection with the double-arcing phenomenon in a cutting torch, J. Appl. Phys. 105: 013309, 2009. 
[6] L. Prevosto, H. Kelly and B. Mancinelli, On the Use of Sweeping Langmuir Probes in Cutting Arc Plasmas Part I: Experimental Results, IEEE Trans. Plasma Sci. 36: 263-270, 2008.

[7] L. Prevosto, H. Kelly and F. O. Minotti, On the Use of Sweeping Langmuir Probes in Cutting Arc Plasmas Part II: Interpretation of the Results, IEEE Trans. Plasma Sci. 36: 271-277, 2008.

[8] Y. P. Raizer, Gas Discharge Physics, Berlin, Germany: Springer, 1991.

[9] L. Prevosto, H. Kelly and B. Mancinelli, On the dynamics of the space-charge layer inside the nozzle of a cutting torch and its relation with the "nondestructive" double-arcing phenomenon, J. Appl. Phys. 110: 083302, 2011.

[10] L. Prevosto, H. Kelly and B. Mancinelli, On the spacecharge boundary layer inside the nozzle of a cutting torch, J. Appl. Phys. 105: 123303, 2009.

[11] M. C. M. van de Sanden, P. P. J. M. Schram. A. G. Peeters, J. A. M. van der Mullen, and G. M. W. Kroesen, Thermodynamic generalization of the Saha equation for a two-temperature plasma, Phys. Rev. A 40: 5273-5276,1989.

[12]R. N. Franklin, You cannot patch active plasma and collisionless sheath, IEEE Trans. Plasma Sci. 30: 352356, 2002.

[13] T. E. Sheridan and J. Goree, Colisional plasma sheath model, Phys. Fluids B 3: 2796-2804, 1991.

[14] S. Ghorui, J. V. R. Heberlein and E. Pfender, Non equilibrium modelling of an oxygen-plasma cutting torch, J. Phys. D: Appl. Phys. 40: 1966-1976, 2007. 